\title{
ORIGINAL ARTICLE \\ Genistein and daidzein induce apoptosis of colon cancer cells by inhibiting the accumulation of lipid droplets
}

\author{
Yu-Si Liang',2, Wen-Tao Qi'*, Weiqun Guo', Chun-Ling Wang'2, Ze-Bin Hu³ and Ai-Ke Li' \\ 'Cereals \& Oils Nutrition Research Group, Academy of State Administration of Grain (ASAG), Beijing, The People's \\ Republic of China; ${ }^{2}$ Key Laboratory of Food Safety and Sanitation, Ministry of Education, College of Food Engineering \\ and Biotechnology, Tianjin University of Science and Technology, Tianjin, The People's Republic of China; ${ }^{3}$ nstitute for In \\ Vitro Diagnostic Reagents Control, The National Institutes for Food and Drug Control (NIFDC), Beijing, The People's \\ Republic of China
}

Abstract

Aim: The purpose of this study was to investigate the possible mechanisms of genistein (GEN) and daidzein (DAI) in inducing apoptosis of colon cancer cells by inhibition of lipid droplets (LDs) accumulation.

Methods: HT-29 cells were used and treated by GEN or DAI in this paper. LDs accumulation was induced and inhibited by oleic acid (OA) and C75, respectively. The expression changes of LDs-related markers were confirmed by semiquantitative real time-PCR (RT-PCR), Western blotting, and immunofluorescence staining.

Results: GEN and DAI effectively reduced the LDs accumulation and downregulated the expression of Perilipin-1, ADRP and Tip-47 family proteins and vimentin levels. GEN and DAI significantly induced the mRNA expression of PPAR- $\gamma, F a s, F A B P$, glycerol-3-phosphate acyltransferase (GPAT3), and microsomal TG transfer protein $(M T T P)$, and reduced the mRNA expression of $U C P 2$. Furthermore, the results showed a decrease of PI3K expression by GEN and DAI when compared with OA treatment, and both GEN and DAI can increase the expression of FOXO3a and caspase- 8 significantly when these proteins were decreased by OA treatment. GEN is more effective than DAI in inducing cell apoptosis.

Conclusion: Our results demonstrated that GEN and DAI inhibit the accumulation of LDs by regulating LDs-related factors and lead to a final apoptosis of colon cancer cells. These results may provide important new insights into the possible molecular mechanisms of isoflavones in anti-obesity and anti-tumor functions.

Keywords: genistein; daidzein; lipid droplets; apoptosis; colon cancer

Received: 30 October 20 I7; Revised: 19 March 20I 8; Accepted: 4 April 20 I8; Published: II May 20 I8

$\mathrm{C}$ olon carcinoma is one of the leading causes of cancer mortality worldwide (1). Increased risk factors such as age, consumption of high-fat diet, lack of physical activities, alcohol, smoking, environmental pollution, and hereditary are recognized as the cause of the colon cancer $(1,2)$. Most of the colon cancers are developed by an accumulation of several mutations throughout the lifetime (3). Furthermore, some incident of colon cancers can be lowered or inhibited by more reasonable life style including healthy diet. Epidemiological studies have suggested that a higher dietary intake of soybean products contributes to a lower incidence of colorectal cancer in Asian countries $(1,2)$.

Soya beans are an abundant source of bioactive compounds of which isoflavonoids have received significant attention due to their potential anticarcinogenic and antiproliferative effects and possible role in many signal transduction pathways $(4,5)$. There are two major kinds of isoflavones in soybeans: glycosides, including daidzin and genistin;and aglycons, including DAI and GEN (6). Dietary aglycons are known to be absorbed faster and metabolized more effectively than glucosides (7). Another study showed that aglycons exerted more beneficial effects on bone metabolism, with positive impacts on human health, than did glucosides (8). Indeed, numerous studies have shown the prominent component of aglycons, GEN, has anticancer properties $(9,10)$. Possible mechanisms mainly include an inhibitory effect on tyrosine kinases and DNA topoisomerases, induction of apoptosis, and modulation of the pathway of signal conduction such as PI3K/Akt and Wnt/ $\beta$-catenin (11). However, the roles of the anticancer effect of aglycons and their molecular targets remain to be further elucidated.

In general, the ability to overcome metabolic stress is a crucial step for cancer cell survival and metastasis (11). It is believed that the accumulation of lipid droplets (LDs) 
offered the required energy that drives cell proliferation during the process and has been considered a hallmark of tumor progression (12). LDs are organelles constitutively present in adipocytes and serve as lipid stores that provide energy for a number of diverse functions in organisms (13-15). Human colon adenocarcinoma cell lines and colon cancer biopsies from patients have been shown to exhibit an increase in adipose differentiation-related protein (ADRP), which is a major structural protein associated with LD (16). Furthermore, inhibition of LD formation has been proved to be correlated with diminished cancer cell proliferation in vitro (17), while the mechanisms involved in this adaptation are still poorly understood $(3,18)$.

In this study, we show the evidence that the regulation of GEN and DAI induces apoptosis of colon cancer cells by inhibiting the accumulation of lipid droplets.

\section{Materials and methods}

\section{Cell culture}

Human colonic cancer HT-29 cells were purchased from the Institute of Biochemistry and Cell Biology, Shanghai Institutes for Biological Sciences, Chinese Academy of Sciences (Shanghai, China), which was originally offered by the American Type Culture Collection (ATCC), Manassas, VA, USA. The cells were grown in Dulbecco's Modified Eagle Medium (Gibco) supplemented with 10\% fetal bovine serum (Gibco). The cells were cultured at $37^{\circ} \mathrm{C}$ in a humidified atmosphere of $95 \%$ air to $5 \% \mathrm{CO}_{2}$.

\section{Cell treatment}

Monolayers of human HT-29 cells were treated with genistein (GEN) and daidzein (DAI purchased from Solarbio (Beijing, China) for $48 \mathrm{~h}$ at concentrations of 25 , $50,100,200$, and $400 \mu \mathrm{M}$, respectively. LDs accumulation was induced with $1 \mathrm{mg} / \mathrm{mL}$ oleic acid (OA) (Sigma, St. Louis, MO, USA). During the treatment, cells were placed in serum-free and antibiotic-free medium.

\section{Cell proliferation}

An inhibitory effect of GEN and DAI on proliferation of colon cancer cell lines was evaluated by the MTT [3-(4,5-dimethylthiazol-2-yl)-2,5-diphenyl tetrazolium] assay. HT-29 cells were plated in 96 -well plates $(5,000$ cells per well). After incubation for $24 \mathrm{~h}$, varied concentrations of GEN and DAI were added into each well, and each concentration was repeated in five wells. After $48 \mathrm{~h}$ incubation, the medium was aspirated, and $0.5 \mu \mathrm{g} / \mathrm{mL}$ MTT was added. Cells were incubated at $37^{\circ} \mathrm{C}$ for another $4 \mathrm{~h}$, and the formed formazan product was solubilized with dimethylsulfoxide (DMSO). The optical density (OD) of each well was then measured on an enzyme-linked immunosorbent assay (ELISA) microplate reader (Multiskan EX, Labsystems, Helsinki, Finland) at $570 \mathrm{~nm}$.
The effects of GEN and DAI on cell proliferation were assessed as percentage cell viability compared to control cells, which were arbitrarily assigned $100 \%$ viability. Each test was performed in triplicate experiments.

\section{Inhibitors}

C75, a fatty acid synthase inhibitor purchased from Sigma-Aldrich Chemical (St. Louis, MO), was used to assess the effect of LDs on protein regulation at a concentration of $50 \mu \mathrm{g} / \mathrm{mL}$.

\section{Scanning electron microscopy analysis}

The cell suspension $\left(1 \times 10^{5} / \mathrm{mL}\right)$ was seeded on cover slips which were partitioned previously into the wells of 6-well plates. After $24 \mathrm{~h}, \mathrm{HT}-29$ cells were treated with $200 \mu \mathrm{M}$ GEN and DAI, respectively, for 24 and $48 \mathrm{~h}$, followed by pre-fixed glutaraldehyde $(2.5 \%)$ at $4^{\circ} \mathrm{C}$ for $1 \mathrm{~h}$. Subsequently, the cells were rinsed thoroughly with phosphate buffered saline (PBS) and post-fixed in $\mathrm{OSO}_{4}(1 \%)$ at $4^{\circ} \mathrm{C}$ for $1 \mathrm{~h}$; after gold spraying, scanning electron microscopy was applied to observe the change of cell surface morphology.

\section{Flow cytometry analysis}

HT-29 cells were seeded in a 6-well plate and treated with $200 \mu \mathrm{M}$ GEN and DAI, respectively, for 24 and $48 \mathrm{~h}$, after which they were collected. Subsequently, the cells were washed with cold PBS and fixed by ethanol $(70 \%$, v/v). The cells were dissolved in PBS (containing PI, RNase, EDTA, and Triton $\mathrm{X}-100, \mathrm{pH} 7.4$ ) and incubated at $37^{\circ} \mathrm{C}$ for $30 \mathrm{~min}$, followed by incubation at $4^{\circ} \mathrm{C}$ for $1 \mathrm{~h}$ in the dark. Ulternately, the samples were detected with a flow cytometry (Becton, Dickinson, San Antonio, TX, USA).

\section{Oil red-O staining}

Monolayers were rinsed three times with PBS, fixed in buffered formalin solution (Sigma) and then stained with Oil Red-O solution (Solarbio, Beijing, China). After rinsing in distilled water, the monolayers were counterstained with Gill's III and mounted with Prolong Gold antifade reagent (Invitrogen, Carlsbad, CA, USA).

\section{Boron-dipyrromethene (BODIPY) staining}

Monolayers were fixed in formalin and incubated with $1 \mu \mathrm{g} / \mathrm{mL}$ BODIPY (ThermoFisher). Slides were washed, mounted with Prolong Gold antifade reagent (Invitrogen, Carlsbad, CA, USA), and observed under fluorescent microscope (Olympus).

\section{Immunofluorescence imaging of vimentin}

Briefly, the cell suspension $\left(1 \times 10^{5} / \mathrm{mL}\right)$ was inoculated on cover slips which were partitioned previously into the wells of a 6-well plate. After $24 \mathrm{~h}, \mathrm{HT}-29$ cells were treated with $200 \mu \mathrm{M}$ GEN and DAI, respectively, for $48 \mathrm{~h}$. Cells were fixed with $3 \%$ formaldehyde in PBS ( $\mathrm{pH}$ 7.4) for 
20 min and washed thrice with PBS. Washed cells were permeabilized using $0.2 \%$ Triton $\mathrm{X}-100$ and blocked in $2 \%$ BSA (B.M.) in PBS. Then the cells were washed thrice with PBS and incubated with the antibody vimentin (dilution $1: 200$ ) with $2 \%$ BSA in PBS at $37^{\circ} \mathrm{C}$ for $1 \mathrm{~h}$. The resulting cells were washed thrice with PBS and incubated with fluorescein isothiocyanate- labeled polyclonal goat anti-mouse IgG antibody (dilution $1: 200$ ) at $37^{\circ} \mathrm{C}$ for $1 \mathrm{~h}$. Cells were stained with propidium iodide (4',6-diamidino2-phenylindole - DAPI) (Sigma) and scanned by laser scanning confocal microscope. All images were acquired using the same intensity and photodetector gain.

\section{DAPI staining}

The levels of nuclear condensation and fragmentation were observed by means of nucleic acid staining with DAPI (Solarbio, Beijing, China). Briefly, HT-29 cells were plated in 6-well plates $\left(10^{5}\right.$ cells per well). After treatment, the cells were washed twice with PBS and fixed with methanol $(\mathrm{MeOH})$ and acetic acid (HAc) $(3: 1, \mathrm{v} / \mathrm{v})$ for $10 \mathrm{~min}$ at $4^{\circ} \mathrm{C}$. The cells were stained with DAPI $(10 \mathrm{mg} / \mathrm{mL})$ for $20 \mathrm{~min}$ in the dark and then observed under a fluorescence microscope (Olympus BX41, Japan) within 15 min.

\section{Protein extraction and immunoblot}

Experimental monolayers were washed with serum-free media, and then total and fractionated proteins were extracted by cell lysis buffer (Cell Signaling Technology, Danvers, MA). The lysates were centrifuged at $12,000 \mathrm{~g}$ for $20 \mathrm{~min}$ at $4^{\circ} \mathrm{C}$. An equal amount of protein after concentration was determined by the Bradford assay (Bio-Rad, Hercules, CA) was loaded on SDS-PAGE and transferred to nitrocellulose membranes (Bio-Rad). After blocking, specific antibodies such as ADRP, FOXO3a, caspase-8, and $\beta$-actin from ABclonal Biotechnology (Wuhan, China); Perilipin-1 from Cell Signaling Technology (Danvers, MA); and vimentin from Abcam (Cambridge, MA) were used to perform detection. Finally, each protein was detected using an enhanced chemiluminescence system (GE Healthcare, Chicago, IL, USA). Blot images were digitized (Chemidoc, Bio-Rad, Milan, Italy), and the area of each band was quantified using the computerized imaging system (QuantityOne, Bio-Rad). Relative OD (arbitrary units) was normalized for control bands in each series and for protein loading (as probed by anti-actin blots). Each test was performed in triplicate experiments.

\section{RNA extraction and semiquantitative RT-PCR procedure}

Total RNA was extracted from cultured HT-29 cells using Trizol reagent according to the manufacture's instruction (TransGen Biotech). RNA concentration was quantified by UV spectrophotometer at 260 and $280 \mathrm{~nm}$. Total RNA was created with first-stand cDNA synthesis kit (TransGen Biotech). Each amplification was performed for 35 cycles, where one cycle profile consisted of denaturation at $94^{\circ} \mathrm{C}$ for $30 \mathrm{~s}$, annealing at $55^{\circ} \mathrm{C}$ for $30 \mathrm{~s}$ and extension at $72^{\circ} \mathrm{C}$ for $120 \mathrm{~s}$. The semiquantitative RT-PCR kit was used to detect mRNA levels of PPAR- $\gamma$, Fas, UCP2, L-FABP, glycerol-3-phosphate acyltransferase (GPAT3), microsomal TG transfer protein (MTTP), and GAPDH according to the manufacturer's instructions. The primer sequences that used for semiquantitative RT-PCR are shown in Table 1.

\section{Statistical analysis}

The experiments were repeated three times, and the mean values were analyzed by a two-tailed unpaired $t$-test. The results were expressed as mean $\pm \mathrm{SD}$. The level of $p<0.05$ was considered to be statistically significant.

\section{Results}

Genistein and daidzein inhibit the proliferation of HT-29 cells The treatment of HT-29 cells by GEN and DAI at 0, 25, $50,100,200$, and $400 \mu \mathrm{M}$ for $48 \mathrm{~h}$ is illustrated in Fig. 1. The results showed that both GEN and DAI inhibited the growth of HT-29 cells remarkably in a dose-dependent manner. The inhibition of proliferation could be up to 46 and $34 \%$ at the concentration of $200 \mu \mathrm{M}$ for GEN and DAI, respectively. Above $200 \mu \mathrm{M}$, no alterations of cell proliferation inhibition were observed with the increase of both GEN and DAI concentrations. Thus, all the treatments of cells in the following experiment were carried out with $200 \mu \mathrm{M}$ GEN or DAI for $48 \mathrm{~h}$.

Table 1. Primer sequences used for semiquantitative RT-PCR

\begin{tabular}{llll}
\hline Gene & Forward & Reverse & Products (bp) \\
\hline PPAR- $\gamma$ & CCTCACAGCTGTTTGCCAAG & GTGTATCAGCAGTTCCACTCAC & 398 \\
Fas & ATTGCTTGTGTTTGTGGGTGC & AGCAATCTCTTTGCTCAGGCT & 315 \\
UCP2 & CTTCTGCACCACTGTCATCG & TCACACTTGGCTGCTACTCAC & 275 \\
L-FABP & AGCGGCTCACATACCCTAAT & TTATGAAGCACCCTGGGCAT & 478 \\
GPAT3 & CACTTTTTAGAGCCAAAAGGAATCT & AAGTCAGCAGACAAGGGTGG & 432 \\
MTTP & AGCACATCCAGGTCACCTCT & CCTGAACGCTGCATCTTACC & 337 \\
GAPDH & GGACTCATGGTATGAGAGCTGG & GATGGCATGGACTGTGGTCT & 227 \\
\hline
\end{tabular}




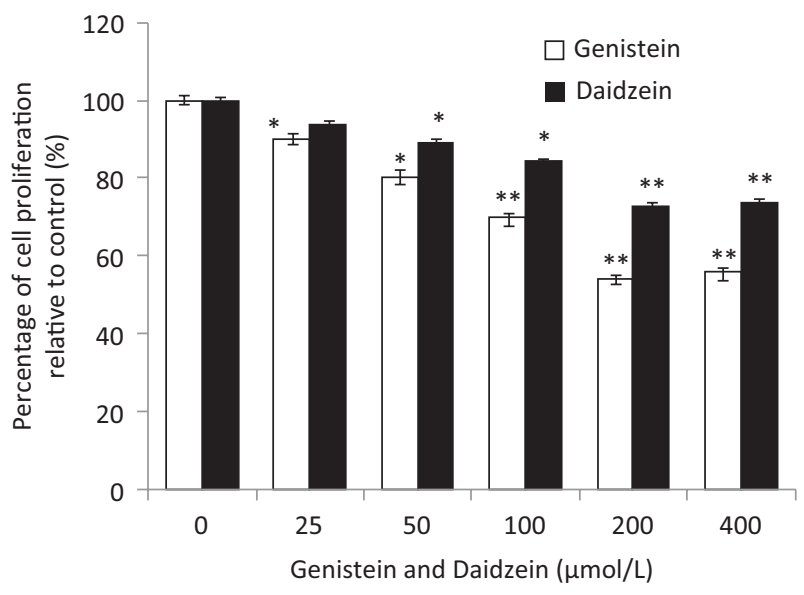

Fig. 1. The effect of GEN and DAI on proliferation of HT-29 cells. HT-29 cells were exposed to GEN and DAI for $48 \mathrm{~h}$. Proliferation was determined by MTT assessed as percentage cell viability compared to control cells, which were arbitrarily assigned $100 \%$ viability. The results were expressed as means $\pm \mathrm{SD}(n=6) .{ }^{*} p<0.05, * * p<0.01$ compared with control cells.

\section{Genistein and daidzein induce apoptosis of HT-29 cells}

Cell apoptosis is a procedure of cell disintegration accompanied by featured morphological variation such as condensation of chromatin, loss of microvilli, blebbing, and apoptotic body formation (19). The morphology changes of HT-29 cells treated with GEN or DAI were observed by a scanning electron microscopy in this article. The results showed that cells with normal volumes and rich microvilli of the control group were kept intact and had integral cell membranes (Fig. 2a), whereas cells treated with GEN or DAI showed typical pre-apoptotic morphologies, characterized by loss of microvilli, cell shrinkage, and blebbing formation at $24 \mathrm{~h}$, and loss of microvilli and appearance of apoptotic bodies were observed significantly at $48 \mathrm{~h}$ (Fig. 2a). The cell-cycle phase distribution and the ratios of apoptotic cells were further determined by flow cytometry with PI staining. The percentage of cells in G1, S, and $\mathrm{G} 2 / \mathrm{M}$ phase was calculated using multi-cycle software. The results showed a significant increase of cells in the G0/G1 phase from $38.48 \pm 5.10 \%$ for control to 51.69 $\pm 5.45 \%$ and $50.76 \pm 4.07 \%(p<0.05)$ for GEN and DAI treatments, respectively, and the apoptotic rate increased significantly ( $p<0.05$ ) from $2.01 \pm 0.15 \%$ for control to $20.49 \pm 8.46 \%$ and $16.11 \pm 5.23 \%$ for GEN and DAI, respectively (Fig. 2b). These results confirmed that both GEN and DAI could inhibit the proliferation of HT-29 cells by induction of apoptosis.

\section{Genistein and daidzein reduced the influences of $O A$ on HT-29 cells}

To determine the effects of GEN and DAI on OA-treated cells, HT-29 cells were loaded with LDs using $1 \mathrm{mg} / \mathrm{mL}$ OA, while the fatty acid synthase inhibitor C75 was used to inhibit the OA-induced LDs accumulation. MTT results showed that OA can induce the proliferation of HT-29 cells $10 \%$ significantly at $48 \mathrm{~h}$ compared to control cells $(p<0.05)$. C75 significantly decreased the proliferation compared to both control and OA-treated cells $(p<0.01)$. The addition of GEN was also found to inhibit the proliferation induced by OA, while the inhibition of DAI was not significant ( $p>0.05$ ) (Fig. 3a). Oil Red-O staining showed that the density of LDs was remarkably increased by OA at $48 \mathrm{~h}$ compared to control cells. The addition of C75 significantly decreased the LDs aggregation during OA treatment. The additions of GEN or DAI were also found at different levels of decrease of LDs accumulation induced by OA similar to the presence of C75 (Fig. 3b).This was confirmed by immunofluorescent staining of neutral lipids (BODIPY) and vimentin (Fig. 3b). Perilipin-1, ADRP and Tip-47 (PAT) proteins, ADRP and perilipin-1, and vimentin were further determined by using Western blotting. It was found that all these proteins were induced significantly by OA addition $(p<0.05)$. While both GEN and DAI can significantly reduce the increased expression of ADRP by 14.6 and $37.5 \%$, respectively, compared with OA-treated cells $(p<0.01)$, the reduction of perilipin-1 was 14.6 and $13.1 \%$, respectively ( $p<0.05$ ) (Fig. 3c). Similar results were found when vimentin expression was analyzed, and the percentage of reduction by GEN and DAI was 21.8 and $13.1 \%$, respectively, compared with OAtreated cells $(p<0.05)$ (Fig. 3c). These results indicate that GEN or DAI can reduce the OA-induced proliferation and effectively reduce the LDs accumulation by downregulating PAT family proteins and vimentin levels in HT-29 cells.

\section{Effects of genistein and daidzein on the mRNA expression of LD-associated genes in HT-29 cells}

In addition to the changes of LDs accumulation and its related proteins, the mRNA expressions of LD-associated genes in the cells were further evaluated using semiquantitative RT-PCR assay. The results showed that OA can significantly induce the mRNA expression of PPAR- $\gamma$, Fas, FABP, GPAT3, and MTTP and reduce the mRNA expression of UCP2 $(p<0.05)$ (Fig. 4). While both GEN and DAI as well as C75 significantly reversed the change of decreased mRNA expression induced by OA $(p<0.05)$ (Fig. 4), these data demonstrate that both GEN and DAI can decrease the LDs density by regulation of the mRNA expression of LD-associated genes in HT-29 cells.

\section{Effects of genistein and daidzein on the expression of PI3K, FOXO3a, and Caspase- 8 proteins}

The results showed a decrease of PI3K expression by GEN and DAI when compared with OA treatment, although the reduction by $\mathrm{C} 75$ and GEN was not remarkable. Both GEN and DAI can increase the expression of FOXO3a 
(A)

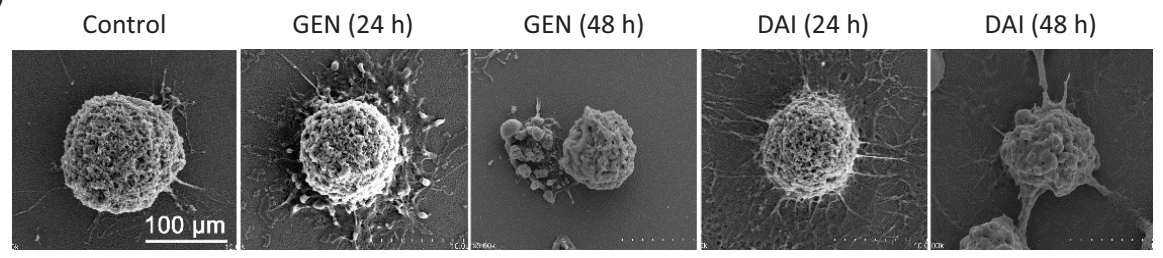

(B)
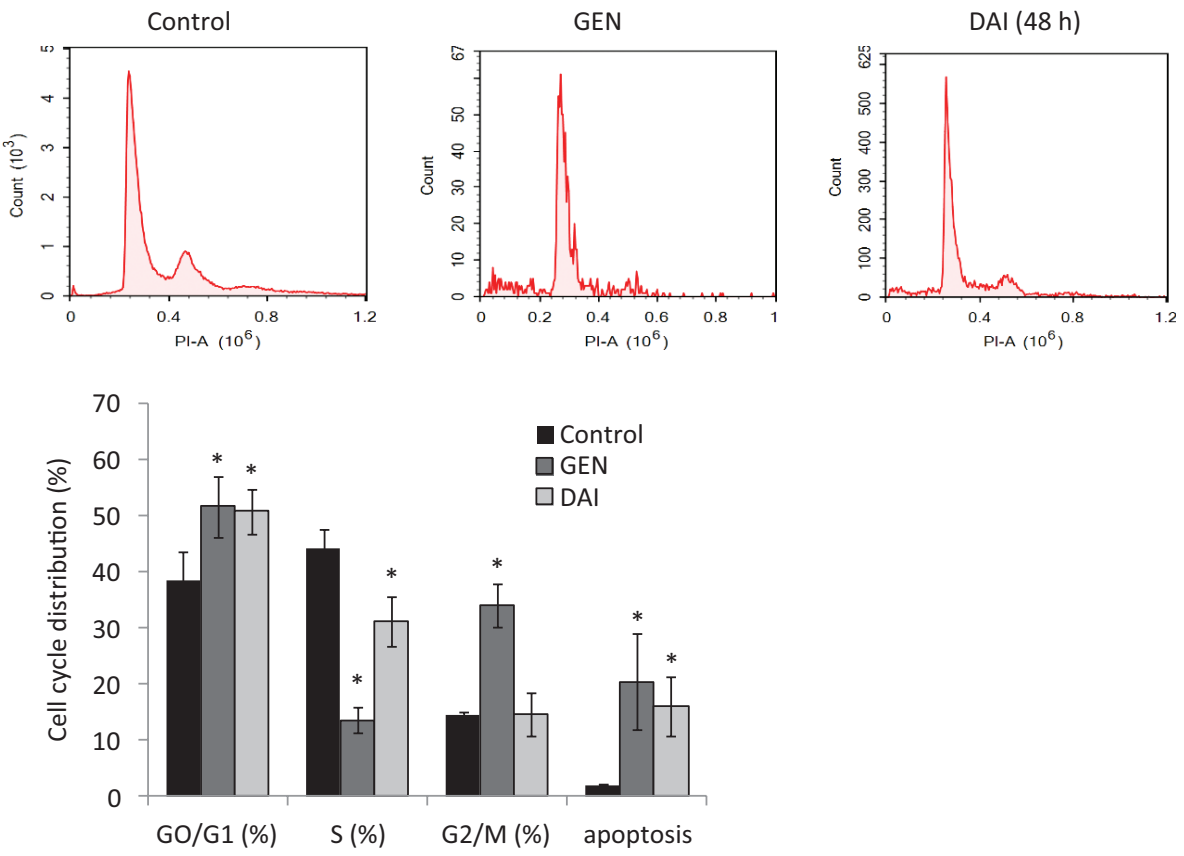

Fig. 2. The apoptosis of HT-29 cells induced by GEN and DAI. (A) Scanning electron micrographs of HT-29 cells after treatment with GEN $(200 \mu \mathrm{M})$ and DAI $(200 \mu \mathrm{M})$, respectively, for 24 and $48 \mathrm{~h}$. Bar $=100 \mu \mathrm{m}$. (B) Cell cycle distribution and apoptosis rate of HT-29 cells by flow cytometry after treatment with GEN $(200 \mu \mathrm{M})$ and DAI $(200 \mu \mathrm{M})$, respectively for $48 \mathrm{~h}$. The results were expressed as means $\pm \mathrm{SD}(n=3) . * p<0.05$ compared with control cells.

and caspase-8 significantly $(p<0.05)$ as well as C75 when these proteins were decreased by OA treatment (Fig. 5). Similar results as GEN and DAI were found when the C75 was added to the HT-29 cells. These results demonstrated that the expressions of FOXO3a and caspase- 8 are negatively associated with LDs accumulation, and all can be upregulated by GEN and DAI in HT-29 cells.

\section{Discussion}

Isoflavones, especially GEN, has been proved to have anticancer properties by many researches (20). This study revealed a novel mechanism that GEN and DAI utilize to inhibit proliferation. GEN and DAI inhibit proliferation and induce apoptosis of colon cancer cells by inhibiting the accumulation of lipid droplets. Upstream, GEN and DAI induced FOXO3 and caspase- 8 activity by targeting PI3k pathway. Downstream, GEN and DAI inhibit OA-induced LDs accumulation by mediated PAT and vimentin proteins via PPAR- $\gamma$ pathway accompanied by the regulation of the mRNA expression of LD-associated genes (Fig. 6).

In the present study, we demonstrated that the exposure of HT-29 cells to GEN and DAI results in a dose-dependent inhibition of cell proliferation and strongly promotes apoptosis. GEN is a more effective inhibitor of cell proliferation than DAI. These results are consistent with several previous studies $(21,22)$.

Several studies have shown increased LD density within cancer cells $(12,23)$, yet the relationship between LDs and the cell cycle is unclear. LDs were previously viewed as storage depots by which colon cancer cells support their high fuel and energy demands during tumor progression $(23,24)$. In the present study, GEN and DAI were found to reduce LDs density and the cell proliferation induced by OA. Furthermore, GEN and DAI were found to decrease the PAT protein family ADRP and perilipin-1. ADRP is a kind of adipocyte factor and an essential protein of lipid metabolism that expresses in the early stage of adipocyte 
(A)

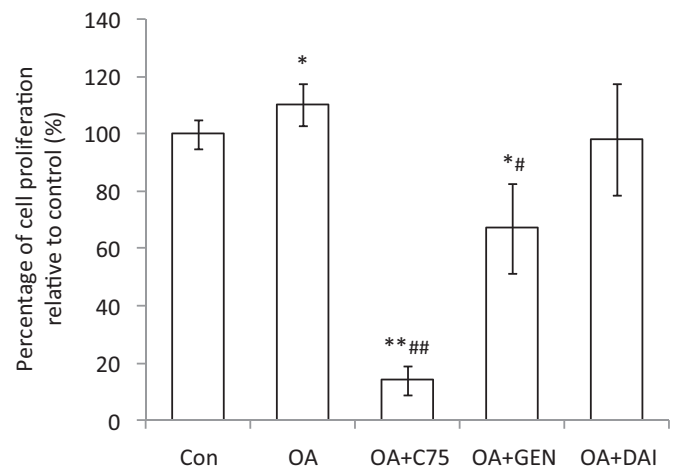

(B)
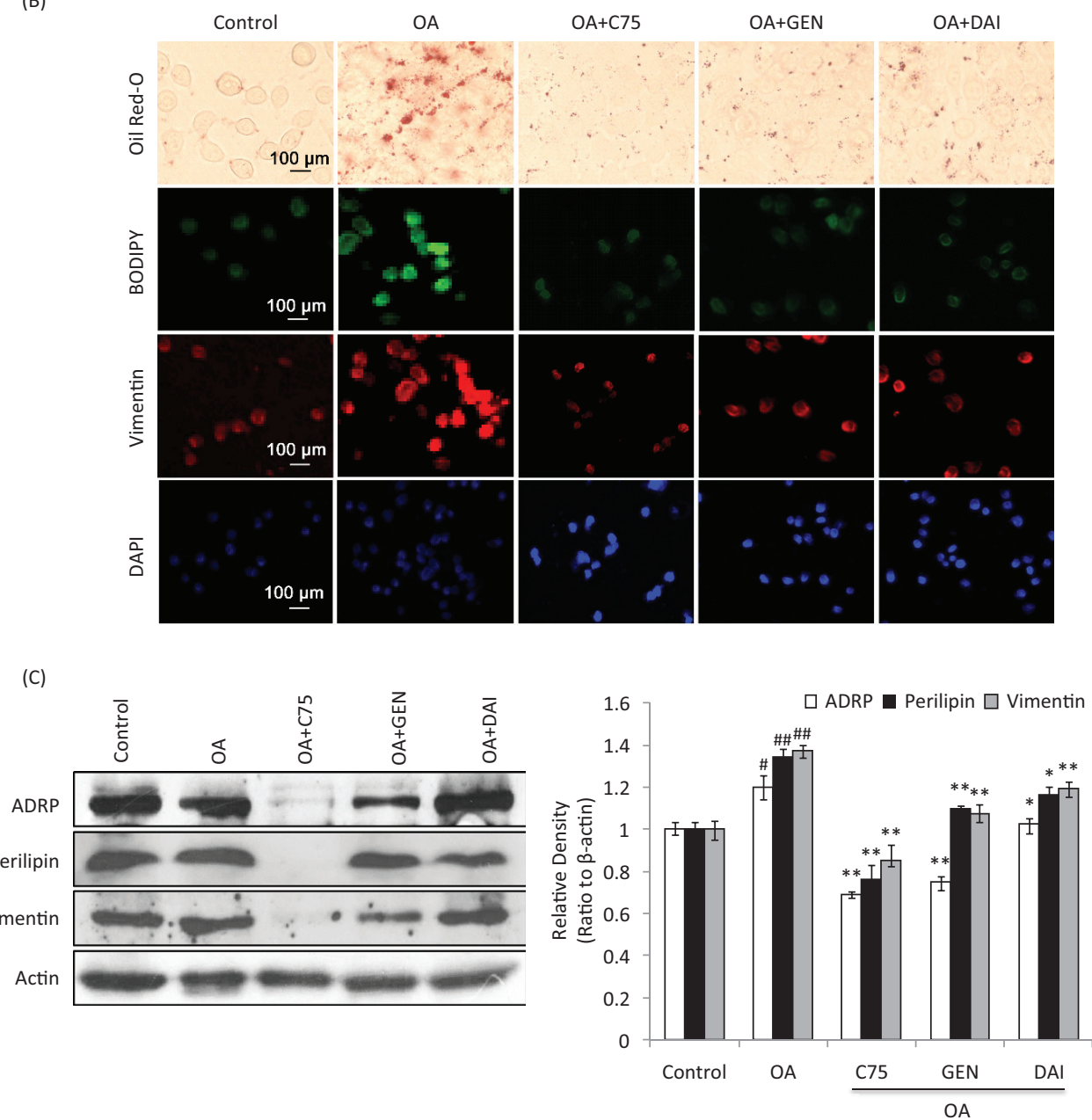

Fig. 3. The effect of GEN and DAI on the proliferation, accumulation of lipid droplets, and the expression of lipid droplets (LDs)-related proteins in HT-29 cells. LDs accumulation was induced with $1 \mathrm{mg} / \mathrm{mL}$ oleic acid (OA) (Sigma). Cells were treated by GEN $(200 \mu \mathrm{M})$ and DAI $(200 \mu \mathrm{M})$, respectively, for $48 \mathrm{~h}$. Fatty acid synthase inhibitor C75 was used to assess the effect of LDs on protein regulation at a concentration of $50 \mu \mathrm{g} / \mathrm{mL}$. (A) Proliferation was determined by MTT assessed as percentage cell viability compared to control cells, which were arbitrarily assigned $100 \%$ viability. The results were expressed as means \pm SD $(n=6)$. \#p $<0.05$, \#\#p $<0.01$ compared with control cells; ${ }^{*} p<0.05, * * p<0.01$ compared with OA-treated cells. (B) LDs density was determined by Oil Red-O and BODIPY staining. The expressions of cytoskeletal proteins, vimentin, were examined by immunofluorescence staining. Morphological evidence of cell apoptosis was measured by DAPI staining. The stained cells were observed under a laser confocal fluorescence microscope $(\times 400)$. Bar $=100 \mu \mathrm{m}$. (C) The expressions of ADRP, perilipin, and vimentin were analyzed by western blot. Density of the bands was quantified by densitometry analysis and was presented after normalization by $\beta$-actin. The results were expressed as means $\pm \mathrm{SD}(n=3) .{ }^{\#} p<0.05,{ }^{\# \#} p<0.01$ compared with control cells; ${ }^{*} p<0.05,{ }^{* *} p<0.01$ compared with OA-treated cells. 

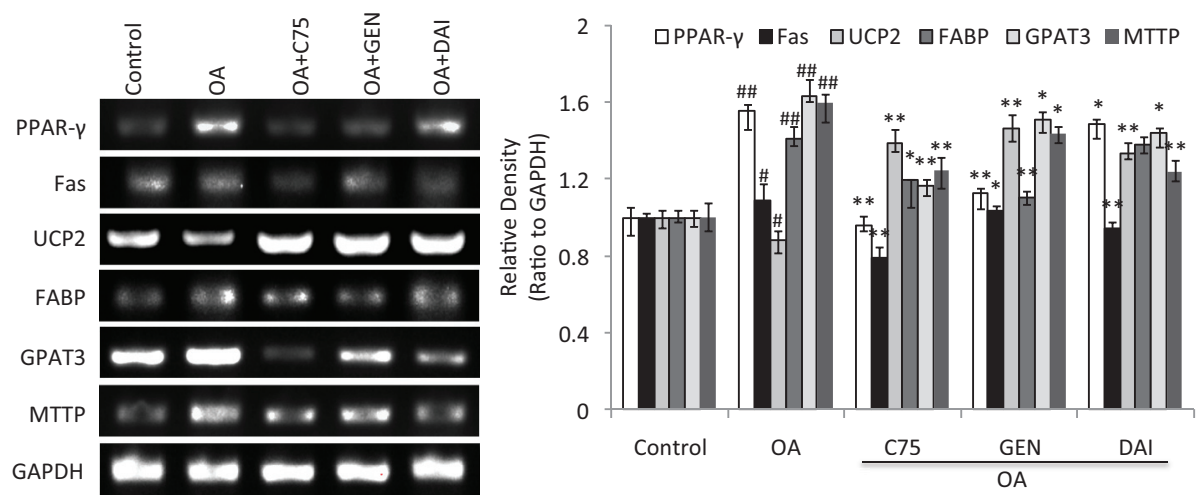

Fig. 4. The effect of GEN and DAI on the mRNA expressions of LD-associated genes in HT-29 cells. mRNA expressions of $P P A R-\gamma, F a s, F A B P, G P A T 3, M T T P$, and UCP2 were determined by RT-PCR analysis. Quantifications of the mRNA were normalized by GAPDH. HT-29 cells were treated with GEN $(200 \mu \mathrm{M})$ and DAI $(200 \mu \mathrm{M})$, respectively, for 48 h. The results were expressed as means $\pm \mathrm{SD}(n=3) .{ }^{\#} p<0.05,{ }^{\# \#} p<0.01$ compared with control cells; $* p<0.05,{ }^{* *} p<0.01$ compared with OA-treated cells.
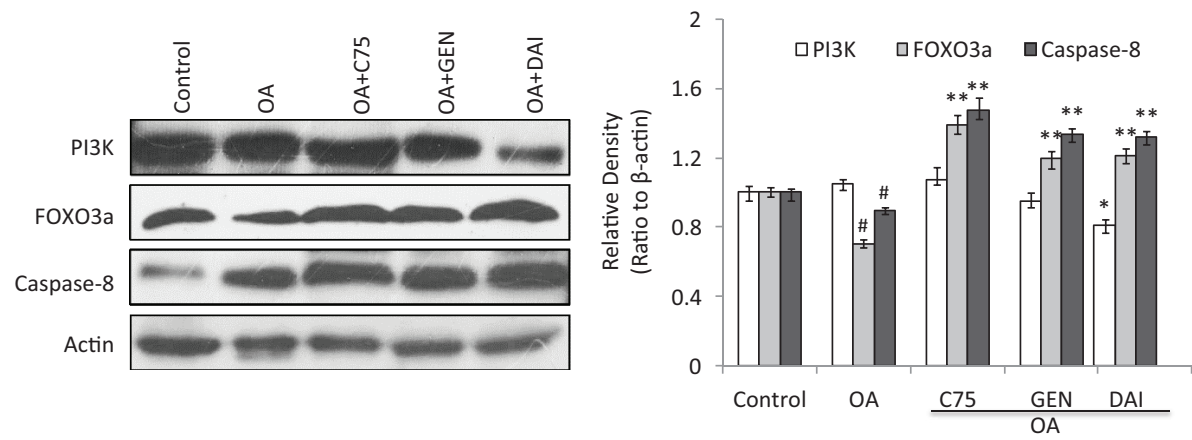

Fig. 5. The effect of GEN and DAI on the expressions of PI3K, FOXO3, and caspase-8. Western blot analysis was carried out to demonstrate the expression of proteins. Densities of the bands were quantified by densitometry analysis. Data are presented after normalization by $\beta$-actin. HT-29 cells were treated by GEN $(200 \mu \mathrm{M})$ and DAI $(200 \mu \mathrm{M})$, respectively, for $48 \mathrm{~h}$. The results were expressed as means $\pm \mathrm{SD}(n=3) .{ }^{*} p<0.05,{ }^{\# \#} p<0.01$ compared with control cells; $*{ }^{*} p<0.05,{ }^{* *} p<0.01$ compared with OA-treated cells.

differentiation; it plays an important role in promoting the formation of lipid droplets (16). Perilipin-1 is a highly phosphorylated adipocyte protein that locates at the surface of the lipid droplet and serves important functions in double-regulating triacylglycerol metabolism (25). Upregulation of LD-associated proteins such as PLIN family, CIDEC, CIDEA, HILPDA, FITM1, FITM2, and G0S2 by PPARs including PPAR $-\gamma$ has been considered a mechanism to link uptake of lipids to regulation of lipid storage capacity (26). In this article, we found a significant decrease of PPAR- $\gamma$ mRNA expression by GEN and DAI, especially GEN. These results demonstrate that the inhibitory effects of GEN and DAI on LDs accumulation are mediated through PPAR- $\gamma$ pathway.

It has been confirmed that the fatty acid synthase (Fas) is an essential component and plays an important role in fatty acid biosynthesis metabolism (27). Increased expression of Fas has been proved to provide a survival advantage to colorectal cancer cells via upregulation of cellular respiration (28). Liver fatty acid binding protein (L-FABP) is an abundant cytosolic fatty acid carrier involved in the long chain FA transport, which can be active by PPAR- $\gamma(29)$. GPAT3 is the rate-limiting enzyme involved in triglycerides (TGs) synthesis, and its overexpression in mammalian cells results in increased TG formation (30). MTTP is a protein required for lipoprotein assembly and secretion, whose gene was found to be strongly associated with fatty acid profile and positively mediated the fat deposits $(31,32)$. All these LDs-promoted factors were found can to be significantly reduced by GEN and DAI. Besides, it is interesting to note that both GEN and DAI significantly increased mRNA levels of the thermogenesis-related protein UCP2 that can improve abnormal lipid metabolism by suppressing lipogenesis and promoting fatty acid (33). The relationship between vimentin and LDs was already described (34). And the specific binding 


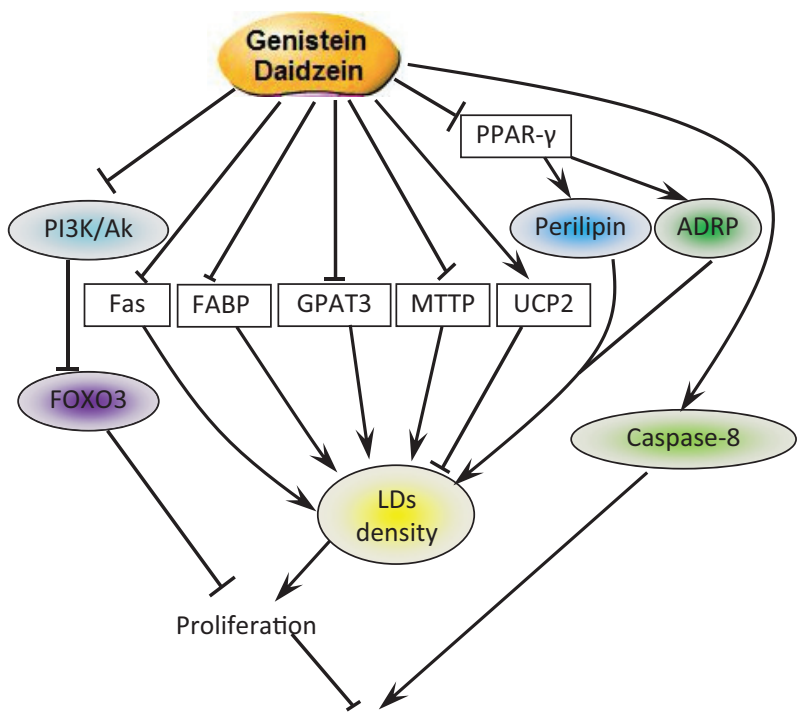

Apoptosis

Fig. 6. Pathways involved in cell apoptosis and LDs decrease induced by GEN and DAI. GEN and DAI inhibit the accumulation of LDs by downregulating the expression of LDs-related proteins perilipin and ADRP combined with the regulations of lipid metabolism-associated genes. On the other hand, GEN and DAI induce caspase- 8 expression and inhibit PI3K activity, which results in an increased level of FOXO3. All these functions lead to a final apoptosis of colon cancer cells.

of perilipin-1 and vimentin were found to protect LDs and against lipase activity in human adipose cells (35). In this article, we found that the level of vimentin promoted by $\mathrm{OA}$ can be significantly decreased by the addition of GEN and DAI. All these results strongly demonstrate that GEN and DAI have a dual function in regulating both cell proliferation and LD-mediated metabolic needs for sustained cell growth.

The effects of GEN and DAI on PI3K, FOXO3, and caspase- 8 expression were further determined. It has been previously demonstrated by several reports that GEN inhibits proliferation in colon cancer cells via PI3K/Akt, a pathway known to be critical to colon cancer progression (36-38). We previously found that GEN-inhibited PI3K/Akt activation leads to inactivation of FOXO3, which negatively regulates proliferation in colon cancer cells (36). Here we confirmed both GEN and DAI significantly increased the level of FOXO3. Furthermore, we found that GEN and DAI significantly increased the caspase- 8 expression that has been known as a key mediator during the executional phase of apoptosis (39). The present study showed that GEN and DAI treatment caused activation of caspase- 8 whose activity was inhibited by OA, suggesting that GEN and DAI induce cell death through a caspase-dependent mechanism.
In summary, our results demonstrated that GEN and DAI inhibit the accumulation of LDs by downregulating the expression of LDs-related proteins, perilipin-1 and ADRP, combined with the regulations of lipid metabolism-associated genes. Moreover, GEN and DAI induce caspase- 8 expression and inhibit PI3K activity, which results in an increased level of FOXO3.A1l these functions lead to a final apoptosis of colon cancer cells. We speculate this possible pathway to demonstrate the GEN and DAI mechanisms of inhibition of LD accumulation and apoptosis in HT-29 cells. These results provide important new insights into the possible molecular mechanisms of isoflavones, in addition to its potential as a novel candidate as an anti-obesity and anti-tumor agent.

\section{Acknowledgments}

This work was supported by the National Natural Science Foundation of China (No. 31471591). We thank the colleagues and collaborators for their help in this work.

\section{Authors' contributions}

LY carried out and design the experiments and participated in the preparation of figures. WT envisioned the study and participated in its design, coordination and final manuscript preparation. GW revised the manuscript and participated in the preparation of figures. WC designed hypotheses and the experiments. $\mathrm{ZH}$ participated in the preparation of the manuscript. AL designed the experiments and offered some experimental materials. All authors read and approved the final version of the manuscript.

\section{Conflict of interest and funding}

The authors have not received any funding or benefits from industry or elsewhere to conduct this study.

\section{References}

1. Rebecca S, Carol D, Ahmedin J. Colorectal cancer statistics. CA Cancer J Clin 2014; 64(2): 104-17.

2. Ferlay J, Soerjomataram I, Dikshit R, et al. Cancer incidence and mortality worldwide: sources, methods and major patterns in GLOBOCAN 2012. Int J Cancer 2015; 136(5): 359-86.

3. Sekar V, Anandasadagopan SK, Ganapasam S. Genistein regulates tumor microenvironment and exhibits anticancer effect in dimethyl hydrazine-induced experimental colon carcinogenesis. BioFactors 2016; 42(6): 623-37.

4. Pavese JM, Farmer RL, Bergan RC, et al. Inhibition of cancer cell invasion and metastasis by genistein. Cancer Metastasis Rev 2010; 29(3): 465-82.

5. Li YR, Yun TT, Liu S, et al. Analysis of water-soluble bioactive compounds in commonly consumed soymilk in China. J Food Compos Anal 2016; 46: 29-35.

6. Lee $\mathrm{CH}$, Yang $\mathrm{L}, \mathrm{Xu} \mathrm{JZ}$, et al. Relative antioxidant activity of soybean isoflavones and their glycosides. Food Chem 2005; 90(4): 735-41. 
7. Cassidy A, Hanley B, Lamuela-Raventos RM, et al. Isoflavones, lignans and stilbenes - origins, metabolism and potential importance to human health. J Sci Food Agric 2000; 80(7): 1044-62.

8. Coward L, Barnes NC, Setchell KDR, et al. Genistein, daidzein, and their. beta.-glycoside conjugates: antitumor isoflavones in soybean foods from American and Asian diets. J Agric Food Chem 1993; 41(11): 1961-7.

9. Spagnuolo C, Russo GL, Orhan IE, et al. Genistein and cancer: current status, challenges, and future directions. Adv Nutr 2015; 6(4): 408-19.

10. Shafiee G, Saidijam M, Tavilani H, et al. Genistein induces apoptosis and inhibits proliferation of HT29 colon cancer cells. Int J Mol Cell Med 2016; 5(3): 178-91.

11. Senft D, Ronai ZE. Adaptive stress responses during tumor metastasis and dormancy. Trends in Cancer 2016; 2(8): 429-42.

12. Qi W, Fitchev PS, Cornwell ML, et al. FOXO3 growth inhibition of colonic cells is dependent on intraepithelial lipid droplet density. J Biol Chem 2013; 288(23): 16274-81.

13. Straub BK, Stoeffel P, Heid H, et al. Differential pattern of lipid droplet-associated proteins and de novo perilipin expression in hepatocyte steatogenesis. Hepatology 2008; 47(6): 1936-46.

14. Straub BK, Herpel E, Singer S, et al. Lipid droplet-associated PAT-proteins show frequent and differential expression in neoplastic steatogenesis. Mod Pathol 2010; 23(3): 480-92.

15. Beilstein F, Carrière V, Leturque $\mathrm{A}$, et al. Characteristics and functions of lipid droplets and associated proteins in enterocytes. Exp Cell Res 2016; 340(2): 172-9.

16. Pfisterer SG, Bakula D, Frickey T, et al. Lipid droplet and early autophagosomal membrane targeting of $\mathrm{Atg} 2 \mathrm{~A}$ and $\mathrm{Atg} 14 \mathrm{~L}$ in human tumor cells. J Lipid Res 2014; 55(7): 1267-78.

17. Accioly MT, Pacheco P, Maya-Monteiro CM, et al. Lipid bodies are reservoirs of cyclooxygenase-2 and sites of prostaglandin-E2 synthesis in colon cancer cells. Cancer Res 2008; 68(6): 1732-40.

18. Londos C, Sztalryd C, Tansey JT, et al. Role of PAT proteins in lipid metabolism. Biochimie 2005; 87(1): 45-9.

19. Dai C, Zhang X, Meng M, et al. Inhibitory effect on HT-29 colon cancer cells of a water-soluble polysaccharide obtained from highland barley. Int J Biol Macromol 2016; 92: 88-95.

20. Sanaei M, Kavoosi F, Atashpour S, et al. Effects of genistein and synergistic action in combination with tamoxifen on the HepG2 human hepatocellular carcinoma cell line. Asian Pac J Cancer Prev 2017; 18(9): 2381-6.

21. Kuntz S, Wenzel U, Daniel H. Comparative analysis of the effects of flavonoids on proliferation, cytotoxicity, and apoptosis in human colon cancer cell lines. Eur J Nutr 1999; 38(3): 133-42.

22. Lepri SR, Zanelatto LC, Da SP, et al. Effects of genistein and daidzein on cell proliferation kinetics in HT29 colon cancer cells: the expression of CTNNBIP1 ( $\beta$-catenin), APC (adenomatous polyposis coli) and BIRC5 (survivin). Hum Cell 2014; 27(2): 78-84.

23. Boström P, Andersson L, Rutberg M, et al. SNARE proteins mediate fusion between cytosolic lipid droplets and are implicated in insulin sensitivity. Nat Cell Biol 2007; 9(11): 1286-93.

24. Liu Q, Luo Q, Halim A, et al. Targeting lipid metabolism of cancer cells: a promising therapeutic strategy for cancer. Cancer Lett 2017; 401: 39-45.

25. Daniel J, Kapoor N, Sirakova T, et al. The perilipin-like PPE15 protein in Mycobacterium tuberculosis is required for triacylglycerol accumulation under dormancy-inducing conditions. Mol Microbiol 2016; 101(5): 784-94.
26. Rodriguez MADLR, Kersten S. Regulation of lipid droplet-associated proteins by peroxisome proliferator-activated receptors. Biochim Biophys Acta 2017; 19: 1388-981.

27. Baenke F, Peck B, Miess H, et al. Hooked on fat: the role of lipid synthesis in cancer metabolism and tumour development. Dis Model Mech 2013; 6(6): 1353-63.

28. Zaytseva YY, Harris JW, Mitov MI, et al. Increased expression of fatty acid synthase provides a survival advantage to colorectal cancer cells via upregulation of cellular respiration. Oncotarget 2015; 6(22): 18891-904.

29. Ong KT, Mashek MT, Davidson NO, et al. Hepatic ATGL mediates PPAR- $\alpha$ signaling and fatty acid channeling through an L-FABP independent mechanism. J Lipid Res 2014; 55(5): 808-15.

30. Pagac M, Cooper D, Qi Y, et al. SEIPIN regulates lipid droplet expansion and adipocyte development by modulating the activity of glycerol-3-phosphate acyltransferase. Cell Rep 2016; 17(6): 1546-59.

31. Herrmann SM, Poirier O, Nicaud V, et al. Identification of two polymorphisms in the promoter of the microsomal triglyceride transfer protein (MTP) gene: lack of association with lipoprotein profiles. J Lipid Res 1998; 39(12): 2432-35.

32. Nadal-Serrano M, Pons DG, Sastre-Serra J, et al. Genistein modulates oxidative stress in breast cancer cell lines according to $E R \alpha / E R \beta$ ratio: effects on mitochondrial functionality, sirtuins, uncoupling protein 2 and antioxidant enzymes. Int $\mathrm{J}$ Biochem Cell Biol 2013; 45(9): 2045-51.

33. Sung YY, Kim DS, Kim SH, et al. Anti-obesity activity, acute toxicity, and chemical constituents of aqueous and ethanol Viola mandshurica extracts. BMC Complement Altern Med 2017; 17(1): 297.

34. Franke WW, Hergt M, Grund C. Rearrangement of the vimentin cytoskeleton during adipose conversion: formation of an intermediate filament cage around lipid globules. Cell 1987; 49(1): 131-41.

35. Heid H, Rickelt S, Zimbelmann R, et al. On the formation of lipid droplets in human adipocytes: the organization of the perilipin-vimentin cortex. PLoS One 2014; 9(2): e90386.

36. Samuels Y, Ericson K. Oncogenic PI3K and its role in cancer. Curr Opin Oncol 2006; 18(1): 77-82.

37. Sheng H, Shao J, Townsend CM Jr, Evers BM. Phosphatidylinositol 3-kinase mediates proliferative signals in intestinal epithelial cells. Gut 2003; 52(10): 1472-8.

38. Kaarin W, Weber CR, Qi W, et al. Genistein inhibits proliferation of colon cancer cells by attenuating a negative effect of epidermal growth factor on tumor suppressor FOXO3 activity. BMC Cancer 2011; 11(1): 219-27.

39. Monie TP, Bryant CE. Caspase-8 functions as a key mediator of inflammation and pro-IL-1 $\beta$ processing via both canonical and non-canonical pathways. Immunol Rev 2015; 265(1): $181-93$.

\footnotetext{
*Wen-Tao Qi

Academy of State Administration of Grain, P.R.C

No. I I Baiwanzhuang Street, Beijing, 100037

The People's Republic of China

Tel: +86 13910473904.

Email: qwt@chinagrain.org
} 